\title{
A Case of Dengue Hemorrhagic Fever and the Use of Supportive Therapy
}

\author{
Aishah Ali, MD, Emily Sutton, MD
}

\section{INTRODUCTION}

Dengue fever is a mosquito-bound viral illness that occurs in tropical climates. It is endemic in 110 countries. ${ }^{1}$ Fifty to three hundred and ninety million people are infected worldwide every year, ${ }^{2}$ leading to $\sim 25,000$ deaths. ${ }^{3}$ It is often an asymptomatic self-limited illness, which can present with myalgias, arthralgias, and hemorrhagic manifestations such as petechiae. In a fraction of cases, dengue fever can proceed to dengue hemorrhagic fever (DHF), which can be life threatening causing thrombocytopenia, bleeding and increased vascular permeability. Dengue shock syndrome (DSS) can then ensue, if hypotension occurs, and is fatal in $>10 \%$ of patients. ${ }^{4}$

Here we present a case of Dengue fever, which proceeded to DHF and finally DSS. Our case demonstrates the supportive nature of treatment and how catastrophic severe dengue fever can be.

\section{CASE PRESENTATION}

A 64 year-old Pakistani female with a history of diabetes mellitus presented to her local hospital with severe body aches and malaise three days after returning from a two month long trip to Pakistan. During her stay, several family members had a self-limited febrile illness and one was diagnosed with dengue fever. On admission she was found to be febrile and thrombocytopenic, raising concern for dengue fever. She rapidly deteriorated with acute renal failure, acute liver failure (Table 1), new onset seizures, and required intubation and mechanical ventilation. She was then transferred to Thomas Jefferson University Hospital where she was emergently started on molecular adsorbent recycling system (MARS) therapy and continuous veno-venous hemodialysis (CVVHD).

\begin{tabular}{|c|c|c|c|}
\hline Lab Parameter & Value (normal range) & Lab Parameter & Value (normal range) \\
\hline AST & $11035 \mathrm{IU} / \mathrm{L}(7-35)$ & Sodium & $138 \mathrm{mmol} / \mathrm{L}(135-146)$ \\
\hline ALT & 1925 IU/L (1 - 30) & Potassium & $3.3 \mathrm{mmol} / \mathrm{L}(3.5-5)$ \\
\hline Total Bilirubin & $6.5 \rightarrow 14.7 \mathrm{mg} / \mathrm{dL}(0.1-0.9)$ & Chloride & $84 \mathrm{mmol} / \mathrm{L}(89$ - 109) \\
\hline Direct Bilirubin & $5.3 \rightarrow 13.2 \mathrm{mg} / \mathrm{dL}(0.0-0.3)$ & Bicarbonate & 13 mmol/L (24 - 32) \\
\hline Alkaline phosphatase & $230 \rightarrow 239$ IU/L (25 - 120) & BUN & 22 mg/dL (7 - 26) \\
\hline GGT & $254 \mathrm{IU} / \mathrm{L}(5-55)$ & Creatinine & $2.3 \mathrm{mg} / \mathrm{dL}(0.7-1.4)$ \\
\hline $\mathrm{LDH}$ & 8490 IU/L (105 - 333) & Glucose & 179 mg/dL (70 - 100) \\
\hline Lactate & $27.6 \mathrm{mmol} / \mathrm{L}(0.5-2.2)$ & Anion Gap & $13 \mathrm{mmol} / \mathrm{L}(4-16)$ \\
\hline PT & 25.9 seconds $(8.6-13.0)$ & WBC & $6.7 \mathrm{~B} / \mathrm{L}(4-11)$ \\
\hline INR & $2.44 \sec (0.79-1.21)$ & Bands & $30 \%(0-9)$ \\
\hline PTT & $39 \mathrm{sec}(28-38)$ & Hemoglobin & $8.4 \mathrm{~g} / \mathrm{dL}(12.5-15)$ \\
\hline Fibrinogen & 180 mg/dL (203 - 451) & Hematocrit & $25.5 \%(36-46)$ \\
\hline D dimer & $2661 \mathrm{ng} / \mathrm{mL}(<331)$ & Platelets & $33 \mathrm{~B} / \mathrm{L}(140-400)$ \\
\hline
\end{tabular}




\section{DIFFERENTIAL DIAGNOSIS}

The patient's recent history of travel suggested an infectious etiology, particularly dengue as it is endemic in Pakistan. In addition, history obtained provided evidence of a local outbreak. Furthermore, her timeline of fevers and thrombocytopenia 3-7 days after her return supported the diagnosis of DHF. The incubation period for dengue is typically 3-14 days. ${ }^{5}$ Congo fever was also considered but was less likely as it isn't commonly seen in the area she visited in Pakistan. As her hospital course progressed and she became more thrombocytopenic with increasing evidence of severe vascular permeability (Figure 1), the diagnosis of DHF became more apparent. In addition, her serum dengue $\lg G$ and $\lg M$ were reported positive several days after admission.

\section{OUTCOME AND FOLLOW-UP}

Although her aminotransferase levels improved rapidly on MARS (Table 2), the patient's hospital course was complicated by seizures and a new large ischemic stroke in the left medial temporal region. After one week of MARS therapy with no improvement in her mental status, she underwent a transjugular liver biopsy that showed $50-75 \%$ necrosis of liver parenchyma. Given her poor prognosis secondary to severe dengue infection, as well as the aforementioned stroke, she was deemed unsuitable for liver transplant. Post-ischemic stroke she did not have focal deficits, but it was difficult to fully neurologically assess her because of unresponsiveness. She was maintained on MARS/CVVHD for one more

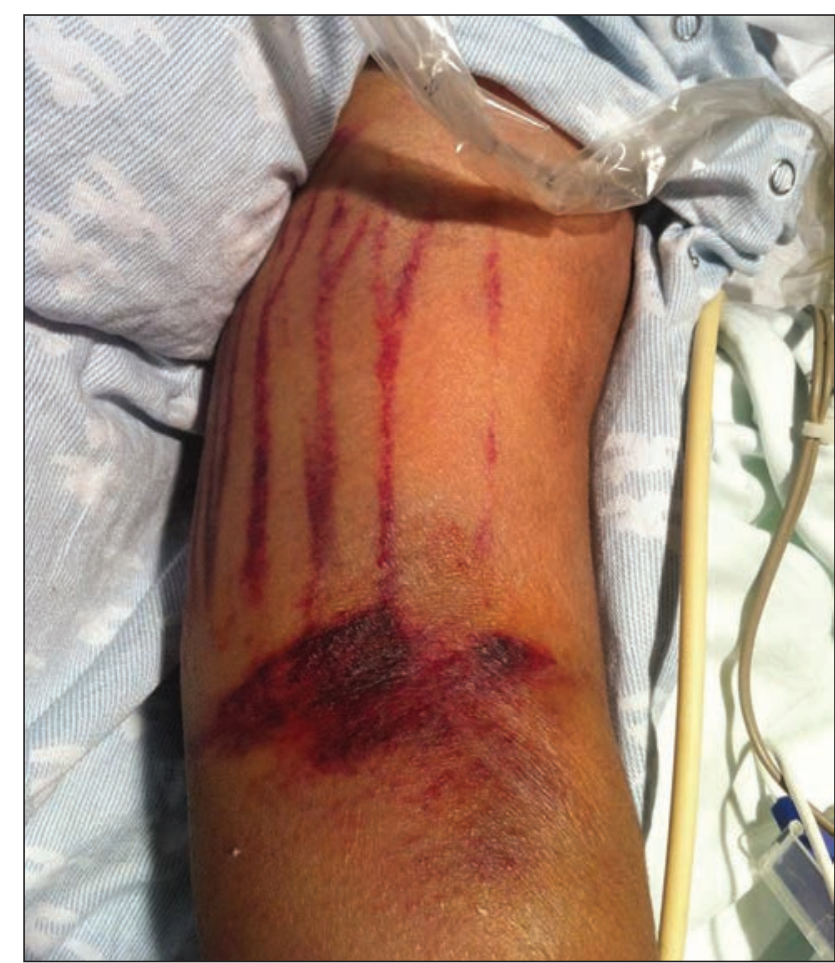

Figure 1. The tourniquet test also known as the capillary-fragility test. Seen in areas of pressure, such as tourniquets or blood pressure cuffs.

week with the hope that longer therapy would improve her outcome. Her hospital course was complicated by tracheobronchitis and septic shock requiring multiple vasopressors. As she still had no sign of neurologic recovery, the family decided to withdraw life support.

Table 2: Lab Values Pre and Post Therapy

\begin{tabular}{l|l|l|}
\hline Lab & Pre-supportive Therapy (normal range) & Post-supportive therapy (normal range) \\
\hline AST & $10705 \mathrm{IU} / \mathrm{L}(7-35)$ & $101 \mathrm{IU} / \mathrm{L}(7-35)$ \\
\hline ALT & $1924 \mathrm{IU} / \mathrm{L}(1-30)$ & $73 \mathrm{IU} / \mathrm{L}(1-30)$ \\
\hline Total Bilirubin & $6.5 \mathrm{mg} / \mathrm{dL}(0.1-0.9)$ & $14.7 \mathrm{mg} / \mathrm{dL}(0.1-0.9)$ \\
\hline Direct Bilirubin & $5.3 \mathrm{mg} / \mathrm{dL}(0.0-0.3)$ & $13.2 \mathrm{mg} / \mathrm{dL}(0.0-0.3)$ \\
\hline Lactate & $24.8 \mathrm{mmol} / \mathrm{L}(0.5-2.2)$ & $7.2 \mathrm{mmol} / \mathrm{L}(0.5-2.2)$ \\
\hline Creatinine & $2.3 \mathrm{mg} / \mathrm{dL}(0.7-1.4)$ & $0.8 \mathrm{mg} / \mathrm{dL}(0.7-1.4)$ \\
\hline $\begin{array}{l}\text { Pre-support therapy: before } \\
\text { Post-supportive therapy: after MARS and CVVHD were initiated }\end{array}$
\end{tabular}




\section{DISCUSSION}

Dengue fever is caused by the dengue virus, which is carried by several species of the mosquito in the genus Aedes. Classic dengue fever is a self-limited febrile illness that may last up to one week. However, it can become more severe and proceed to the hemorrhagic form where profound increases in vascular permeability can lead to shock and possibly multi-organ failure. The mechanism behind the vascular permeability is unknown but is thought to be secondary to the overwhelming inflammatory response to the virus. ${ }^{6}$

The treatment for dengue is entirely supportive, consisting of aggressive fluid resuscitation. MARS and CVVHD therapy may also be used for life threatening sequelae with potential improvement of acute liver failure, acute kidney failure, and disseminated intravascular coagulation. ${ }^{5}$

There is little evidence regarding the use of MARS in the setting of dengue fever. One case report demonstrated rapid improvement in a patient with dengue-associated hepatic encephalopathy and fulminant hepatic failure after treatment with MARS and CVVHD.? The patient's transaminitis rapidly corrected with MARS, with associated resolution of hepatic encepathopathy. The length of MARS with concurrent CVVHD in this case was three days. In our patient, MARS therapy corrected the transaminitis but did not affect her mental status. It was also surprising that her bilirubin levels remained elevated despite MARS which usually removes albumin-based substances such as bilirubin and bile acids. ${ }^{8}$ It was unclear if her altered mental status was secondary to hepatic encephalopathy, stroke, uremia, or infection.

In hindsight, MARS may not have been indicated in this patient for an additional week after no clinical improvement in the first few days. There are no clear guidelines on the use of MARS or hemodialysis in a patient with acute liver failure. In one study evaluating the improvement in mental status in patients with acute liver failure and encephalopathy who were placed on hemodialysis, $61.5 \%$ had either total $(43.6 \%)$ or partial $(17.9 \%)$ regaining of consciousness. ${ }^{9}$ Comparisons have been made between MARS in combination with continuous veno-venous hemodiafiltration (CVVHDF), CVVHDF alone, and single-pass albumin dialysis (SPAD).? All three forms are similar because they remove some type of waste product from the circulation. SPAD uses a standard renal replacement machine with an albumin dialysate solution and no additional recirculation pump whereas MARS uses standard dialysis solution, an albumin solution, and an additional recirculation pump. MARS and SPAD were both efficient in vitro at removing albumin-bound substances, such as bilirubin; SPAD was actually superior to MARS in removing bile acid waste. Both SPAD and CVVHDF were better than MARS in taking out water-soluble solutes such as ammonia. The most notable difference was that SPAD cost significantly less than MARS; for a seven-hour session, SPAD cost $\$ 810$ whereas MARS cost $\$ 2641$. In our patient, MARS was an expensive treatment to continue without any proven correlation between prolonged length of use and improvement in mortality. Cost-effectiveness must be taken into consideration when alternate therapies are available for fractions of the cost.

As an adjuvant therapy for her fulminant hepatic failure, our patient also received eight days of $\mathrm{N}$-acetylcysteine (NAC). NAC has been shown to be beneficial in patients when liver transplant is not available for non-acetaminophen associated acute liver failure. In a retrospective analysis of eight patients with dengue-associated acute liver failure, NAC was shown to be beneficial in early stages of liver failure but not in advanced stages. ${ }^{10}$ The patient in this analysis had a maximum length of NAC use of 72 hours. No analysis exists comparing MARS versus NAC, or using both treatments, for improved outcomes in acute liver failure.

\section{KEY POINTS}

The treatment for dengue hemorrhagic fever is entirely supportive. Guidelines for the length of MARS treatment in dengue patients in fulminant hepatic failure needs to be further evaluated, as MARS is an expensive treatment only available at a few tertiary level centers. 


\section{REFERENCES}

1. Ranjit S, Kissoon N. Dengue hemorrhagic fever and shock syndromes. Pediatr. Crit. Care Med. 2011; 12 (1): 90-100.

2. Bhatt S, Gething PW, Brady OJ, et al. The global distribution and burden of dengue. Nature.2013; 496 (7446): 504-7.

3. Varatharaj A. Encephalitis in the clinical spectrum of dengue infection. Neurol. India. 2010; 58 (4): 585-91.

4. CDC: Dengue and dengue hemorrhagic fever, information for health care practitioners. http://www.cdph.ca.gov/Healthlnfo/ discond/Documents/DenguelnfoForHealthCarePractitioners.pdf

5. Gubler DJ. Dengue viruses. Desk Encyclopedia of Human and Medical Virology. Boston: Academic Press. 2010; 372-82.

6. Atanu Basu1 \& Umesh C. Chaturvedi. Vascular endothelium: the battlefield of dengue viruses. FEMS Immunol Med Microbiol. 2008; 53 287-299.
7. Penafiel et al. Use of molecular adsorbent recirculating system in acute liver failure attributable to dengue hemorrhagic fever. J Intensive Care Med. 2006; 21(6): 369-71.

8. Sauer IM, Goetz M, Steffen I, et al. In vitro comparison of the molecular adsorbent recirculation system (MARS) and single-pass albumin dialysis (SPAD). Hepatology. 2004; 39: 1408-1414.

9. Denis J, Opolon P, Nusinovici V, Granger A, Darnis F. Treatment of encephalopathy during fulminant hepatic failure by haemodialysis with high-permeability membrane. Gut. 1978; 19: 787-793.

10. Ravindu S. Kumarasena et al. Intravenous $\mathrm{N}$-acetylcysteine in dengue-associated acute liver failure. Hepatol Int. 2010; 4(2): 533-534 